\title{
Einstein-Cartan-Maxwell Theory with Scalar Field through a Five-Dimensional Unification
}

\author{
Christopher KohleR* \\ Fachrichtung Theoretische Physik, Universität des Saarlandes, \\ Postfach 151150, D-66041 Saarbrücken, Germany
}

(October 3, 2018)

\begin{abstract}
A modification of Kaluza-Klein theory is proposed in which, as a result of a symmetry breaking, five-dimensional space-time is partially parallelized implying the appearance of torsion fields. A naturally chosen action functional leads to the Einstein-Cartan-Maxwell theory where the electromagnetic field strength is represented by the fifth component of the torsion 2-form. Incorporation of a scalar field reveals that four-dimensional space-time torsion is not induced by the scalar field.
\end{abstract}

PACS numbers: 04.50.+h, 02.40.-k

\section{INTRODUCTION}

The traditional Kaluza-Klein models [1,2] are restricted to Riemannian geometry of fivedimensional (5-d) space-time in that the condition of vanishing torsion is imposed a priori. In view of the various generalizations of Einstein's general theory of relativity to non-Riemannian space-time geometries (see Ref. [3] for a review), it is a natural task to investigate higher dimensional unifications based on such alternative theories of gravitation. The incorporation of torsion degrees of freedom is the most important modification. Several authors have already studied Kaluza-Klein models in space-times with torsion using, e.g., 5-d Riemann-Cartan space [1 7], 5-d teleparallel space [8,9], or special definitions of the space-time geometry [10 13]. In this article, we address ourselves to the problem of deriving pure Einstein-Cartan-Maxwell theory from a 5-d gravitation within a closed formalism.

We arrive at this goal by means of the imposition of constraints on the 5-d space-time connection which are suggested by the local symmetries of the geometry: 5-d gravitation possesses local $S O(4,1)$ symmetry whereas the dimensionally reduced gravitation has only local $S O(3,1)$ symmetry of the external space-time. The dimensional reduction thus involves a symmetry breaking. The basic idea of this article is to break the symmetry before the dimensional reduction - on the kinematical level.

In section 2, we will show that this approach is consistent with two restrictions on the 5-d connection: (i) The normalized Killing vector field along the internal $S^{1}$-manifold is a parallel vector field with respect to the 5 -d connection. (ii) The parallel transport around the $S^{1}$-manifold is integrable.

Both restrictions concern the distant parallelism of space-time and necessarily imply the appearance of torsion fields. Since the parallel transport in 4-d space-time is not restricted, these geometries shall be referred to as semi-teleparallel.

In section 3 of this article, we construct an action functional for semi-teleparallel geometries. The choice of the action is a natural one in the sense that the construction leads in the extreme cases of pure Riemann-Cartan geometry and teleparallel geometry to the standard actions usually employed.

The subject of section 4 is the application of the chosen action functional to the semi-teleparallel Kaluza-Klein geometry without scalar field. After dimensional reduction, the Einstein-CartanMaxwell theory is exactly reproduced.

*e-mail: c.kohler@rz.uni-sb.de 
In section 5, we investigate the incorporation of a scalar field into the semi-teleparallel KaluzaKlein model. In contrast to the usual approaches to scalar-tensor gravity with torsion, we do not obtain the result that space-time torsion is induced by the scalar field.

The final section contains a discussion of the approach presented in this article.

\section{GEOMETRICAL FRAMEWORK}

In this section, we establish the basic properties of a semi-teleparallel geometry. We assume the 5 -d space-time manifold in Kaluza-Klein theory to have a direct product topology $\mathcal{M} \times S^{1}$ where $\mathcal{M}$ is the external 4-d space-time manifold and the circle $S^{1}$ is the internal manifold. We further assume that on $\mathcal{M} \times S^{1}$ a pseudo-Riemannian metric $\gamma$ of signature $(-++++)$ is defined which admits a spacelike Killing vector field $\boldsymbol{\xi}$ the integral curves of which are the $S^{1}$-manifolds.

Kaluza-Klein theory starts with general relativity on $\mathcal{M} \times S^{1}$. The corresponding action functional possesses local $S O(4,1)$ invariance. The dimensionally reduced action, however, has only local $S O(3,1)$ invariance on $\mathcal{M}$, besides the electromagnetic $U(1)$ invariance. In this article, we modify Kaluza-Klein theory in that we formulate a theory of gravitation on $\mathcal{M} \times S^{1}$ that possesses local $S O(3,1)$ invariance defined on $\mathcal{M}$ from the outset. The corresponding space-time geometry can be obtained through a symmetry breaking as described in the following.

We first consider a linear connection on $\mathcal{M} \times S^{1}$. It is given by a $g l(5, \mathbf{R})$-valued connection 1 -form [14]

$$
\boldsymbol{\omega}=\left(e_{M}^{A} \partial_{P} e_{B}^{M}+e_{M}^{A} e_{B}^{N} \Gamma_{N P}^{M}\right) \mathrm{d} x^{P} E_{A}^{B}
$$

Here, $e_{A}^{M}$ are the components of the frame $e_{A}=e_{A}^{M} \partial_{M}$ where $A, B, \ldots=0,1,2,3,5$ are frame indices and $M, N, \ldots=0,1,2,3,5$ are indices of a local coordinate system $x^{M}$. (Explicit indices in parentheses will be frame indices in the following.) $e_{M}^{A}$ is the inverse matrix of $e_{A}^{M}$ forming the cobasis $e^{A}=e_{M}^{A} \mathrm{~d} x^{M}$. The functions $\Gamma_{N P}^{M}$ in Eq. (2.1) are the Christoffel symbols and $E_{B}^{A}$ are the generators of $G L(5, \mathbf{R})$ satisfying the Lie algebra

$$
\left[E_{B}^{A}, E_{D}^{C}\right]=\delta_{D}^{A} E_{B}^{C}-\delta_{B}^{C} E_{D}^{A}
$$

The metric $\gamma$ on $\mathcal{M} \times S^{1}$ defines an equivalence class of orthonormal frames where $\gamma_{M N}=$ $e_{M}^{A} e_{N}^{B} \eta_{A B}$ with the 5 -d Minkowski metric $\eta_{A B}=\operatorname{diag}(-1,1,1,1,1)$. If the connection 1-form (2.1) is restricted to such frames, we can perform the following decomposition:

$$
\boldsymbol{\omega}=-\frac{1}{2} e_{A}^{M} e_{B}^{N} \mathrm{D}_{P} \gamma_{M N} \mathrm{~d} x^{P} E^{(A B)}+\left(e_{A M} \partial_{P} e_{B}^{M}+e_{A M} e_{B}^{N} \Gamma_{N P}^{M}\right) \mathrm{d} x^{P} E^{[A B]},
$$

where $\mathrm{D}_{M}$ represents the covariant derivative with respect to $\Gamma_{N P}^{M}$ and frame indices are raised and lowered using $\eta_{A B}$. The generators $E^{(A B)}$ and $E^{[A B]}$ are the symmetric and antisymmetric part of $E^{A B} \equiv \eta^{A C} E_{C}^{B}$, respectively. $\mathrm{D}_{P} \gamma_{M N}$ is the well known tensor of nonmetricity the vanishing of which is equivalent to the reduction of $\boldsymbol{\omega}$ to the Lorentz connection

$$
\boldsymbol{\omega}=\frac{1}{2} \omega_{A B} J^{A B}=\frac{1}{2}\left(e_{A M} \partial_{P} e_{B}^{M}+e_{A M} e_{B}^{N} \Gamma_{N P}^{M}\right) \mathrm{d} x^{P} J^{A B},
$$

where $J^{A B} \equiv 2 E^{[A B]}$ are the generators of $S O(4,1)$. The geometric interpretation of the condition $\mathrm{D}_{P} \gamma_{M N}=0$ is that the parallel transport of $\gamma$ is integrable, that is, $\gamma$ is a parallel tensor field.

In Kaluza-Klein theory, it is not sufficient to have a metric on $\mathcal{M} \times S^{1}$; the existence of the Killing vector $\boldsymbol{\xi}$ gives a further constraint on the geometry. We shall treat the Killing vector on an equal footing with the metric $\gamma$ in the sense that we use the Killing vector to reduce the $S O(4,1)$ connection (2.3) to an $S O(3,1)$ connection in the same way as the metric $\gamma$ was used to reduce the $G L(5, \mathbf{R})$ connection (2.1) to the $S O(4,1)$ connection (2.3). For that, we note that the Killing vector $\boldsymbol{\xi}$, together with the metric $\boldsymbol{\gamma}$, defines a restricted equivalence class of orthonormal frames by requiring that $e_{(5)}$ lies in the direction of $\boldsymbol{\xi}$, that is, $e_{(5)}$ is the normalized Killing vector. Using these frames, Eq. (2.3) can be decomposed as 


$$
\boldsymbol{\omega}=e_{a M} \mathrm{D}_{P} e_{(5)}^{M} \mathrm{~d} x^{P} J^{a 5}+\frac{1}{2}\left(e_{a M} \partial_{P} e_{b}^{M}+e_{a M} e_{b}^{N} \Gamma_{N P}^{M}\right) \mathrm{d} x^{P} J^{a b},
$$

where $a, b, \ldots=0,1,2,3$. If the connection is such that $\omega_{a(5) P}=e_{a M} \mathrm{D}_{P} e_{(5)}^{M}$ vanishes, which is equivalent to

$$
\mathrm{D}_{P} e_{(5)}^{M}=0
$$

then the connection reduces to the $S O(3,1)$ connection

$$
\boldsymbol{\omega}=\frac{1}{2}\left(e_{a M} \partial_{P} e_{b}^{M}+e_{a M} e_{b}^{N} \Gamma_{N P}^{M}\right) \mathrm{d} x^{P} J^{a b} .
$$

The condition $(2.5)$ means that $e_{(5)}$ is a parallel vector field, that is, the internal circles represent autoparallels.

While the vanishing of the nonmetricity can always be achieved by choosing the connection to be the Levi-Civita connection with respect to $\gamma$, the condition (2.5) can in general only be fullfilled if the connection possesses torsion. In this case, however, we must further restrict the geometry by requiring that the Killing vector be an infinitesimal affine transformation [14]. This is a natural requirement since it implies that - as in Kaluza-Klein theory — the Christoffel symbols are independent of a coordinate on $S^{1}$, which shall be $\theta$ with range $[0,1]$ in the following. $\left(x^{\mu}\right.$, $\mu=0,1,2,3$, will be coordinates on $\mathcal{M}$.) The Killing vector field is then given by $\boldsymbol{\xi}=\partial_{\theta}$.

Although Eq. (2.6) represents an $S O(3,1)$ connection, the symmetry breaking is not yet completed since we still have the freedom to perform local $\theta$-dependent $S O(3,1)$ transformations of the frames. In order to remove this symmetry, we single out frames $e_{a}$ that are parallel along $S^{1}$, that is, the connection is such that $\omega_{a b 5}=0$ in these frames. The fact that the Christoffel symbols do not depend on $\theta$ and the frames have to be single-valued on $S^{1}$ imposes strong restrictions on such frames. The simplest choice are $\theta$-independent frames. These are determined up to local $S O(3,1)$ transformations on $\mathcal{M}$, which is the required symmetry.

To summarize, we have shown that the breaking of the local $S O(4,1)$ symmetry on $\mathcal{M} \times S^{1}$ down to a local $S O(3,1)$ symmetry that is defined on $\mathcal{M}$ specifies a connection for which the components $\omega^{a}{ }_{(5) M}$ and $\omega^{a}{ }_{b 5}$ of the connection 1-form vanish in a basis where $e_{(5)}$ is the normalized Killing vector field and the basis vectors $e_{a}$ do not depend on $\theta$. Hence, in this frame only the components $\omega^{a}{ }_{b \mu}$ are nonvanishing. This means that, first, the parallel transport of a vector pointing in the fifth dimension is integrable and, secondly, the parallel transport of an arbitrary vector along the fifth dimension is integrable. Since the parallel transport does not depend on $\theta$, this implies that the components $R^{(5) a}{ }_{M N}$ and $R^{a b}{ }_{5 \mu}$ of the curvature 2 -form, defined by $R^{A B}=\mathrm{d} \omega^{A B}+\omega^{A}{ }_{C} \wedge \omega^{C B}$, vanish. This geometry is similar to the teleparallel (or Weitzenböck) geometry. However, the parallelization is performed here only partially justifying the term semi-teleparallel geometry. The corresponding connection also appears, as a special case, in Ref. [10].

\section{ACTION FUNCTIONAL}

The purpose of this section is to construct an appropriate action functional describing the dynamics of the semi-teleparallel geometry. This action should be as close as possible to the usual gravitational actions. Since the semi-teleparallel geometry lies "in between" the pure Riemann-Cartan and the teleparallel geometry, we seek an action which "interpolates" those of the Einstein-Cartan and the teleparallel gravitation.

Our starting point is the action for the Einstein-Cartan gravitation in five dimensions,

$$
\tilde{S}\left[\tilde{e}_{M}^{A}, \tilde{\omega}^{A}{ }_{B M}\right]=\int_{\mathcal{M} \times S^{1}} \mathrm{~d}^{5} x \sqrt{-\gamma} \tilde{R}
$$

where $\gamma$ is the determinant of the metric tensor $\gamma_{M N}$ and $\tilde{R}=\tilde{R}^{A B}{ }_{A B}$ is the 5 -d scalar curvature. The tildes in (3.1) indicate that the respective quantities are not yet those of the semi-teleparallel geometry. A possible procedure for obtaining an action for the teleparallel gravitation consists in the replacement of the components $\tilde{\omega}_{B M}^{A}$ in (3.1) by the Levi-Civita connection components 
$\stackrel{\circ}{\omega}_{B M}^{A}$ and in the subsequent interpretation of the frame $\tilde{e}_{M}^{A}$ as a teleparallel frame $e_{M}^{A}$, that is, the connection components $\omega_{B M}^{A}$ are zero. The resulting action is equivalent to the Einstein action, the underlying geometry, however, is different from the one of general relativity.

The teleparallel geometry corresponds to a reduction of a Lorentz connection to a $\{1\}$ connection. The fact that the semi-teleparallel geometry corresponds to a reduction of an $S O(4,1)$ connection to an $S O(3,1)$ connection suggests that in order to construct an action one should replace only the components $\tilde{\omega}_{(5) M}^{a}$ by the Levi-Civita connection components $\stackrel{\circ}{\omega}^{a}(5) M$. The geometry must then be interpreted as a semi-teleparallel geometry, that is, $\tilde{e}_{M}^{A}$ is a semi-teleparallel frame $e_{M}^{A}$ with $\omega^{a}{ }_{(5) M}=0=\omega^{a} b 5$. Using the general relation

$$
R=\stackrel{\circ}{R}+\frac{1}{4} T^{A B C} T_{A B C}+\frac{1}{2} T^{A B C} T_{C B A}+T^{B}{ }_{B A} T^{C A}{ }_{C}-2 \stackrel{\circ}{D}_{A} T^{B}{ }_{B}^{A}
$$

between the full scalar curvature $R$, the Riemannian scalar curvature $\stackrel{\circ}{R}$, and the torsion tensor $T^{A}{ }_{B C}$ defined by $T^{A}=\mathrm{d} e^{A}+\omega^{A}{ }_{B} \wedge e^{B}$, we obtain, as a result of these substitutions, an action that no longer depends on $\omega^{a}{ }_{b 5}$ and $\omega^{a}{ }_{(5) M}$ :

$$
S\left[e_{M}^{A}, \omega^{a}{ }_{b \mu}\right]=\int_{\mathcal{M} \times S^{1}} \mathrm{~d}^{5} x \sqrt{-\gamma}\left(\stackrel{\circ}{R}+\frac{1}{4} T^{a b c} T_{a b c}+\frac{1}{2} T^{a b c} T_{c b a}+T_{b a}^{b} T^{c a}{ }_{c}\right),
$$

where a surface term in the integrand has been dropped.

The functional (3.3) is the action we will use for the semi-teleparallel formulation of the KaluzaKlein model. The action can be written in a more suitable form if we eliminate $\stackrel{\circ}{R}$ in $(3.3)$ with the help of Eq. (3.2) yielding

$$
S\left[e_{M}^{A}, \omega^{a}{ }_{b \mu}\right]=\int_{\mathcal{M} \times S^{1}} \mathrm{~d}^{5} x \sqrt{-\gamma}\left({ }^{(4)} R-\frac{1}{4} T^{(5) a b} T_{(5) a b}-2 T^{(5)}{ }_{(5) a} T^{b a}{ }_{b}\right),
$$

where we have replaced $R$ by the 4 -d scalar curvature ${ }^{(4)} R=R^{a b}{ }_{a b}$ which is possible because the components $R^{a(5)}$ of the curvature 2 -form vanish. Moreover, we have discarded the divergence appearing in Eq. (3.2) which leads to a surface term in (3.4).

\section{EINSTEIN-CARTAN-MAXWELL THEORY}

In order to derive from the action (3.4) an effective 4-d action through dimensional reduction, we have to specify the basis $e_{A}$. A natural choice for $e_{(5)}$ is $e_{(5)}=\partial_{\theta}$. As for $e_{a}$, we make the ansatz $e_{a}=e_{a}^{\mu}\left(\partial_{\mu}-A_{\mu} \partial_{\theta}\right)$ where $e_{a}^{\mu}$ and $A_{\mu}$ are functions on $\mathcal{M}$. The corresponding cobasis is

$$
e^{a}=e_{\mu}^{a} \mathrm{~d} x^{\mu}, \quad e^{(5)}=\mathrm{d} \theta+A_{\mu} \mathrm{d} x^{\mu} .
$$

The parameterization of this cobasis leads to the metric usually employed in the Kaluza-Klein model,

$$
\gamma_{M N}=\left(\begin{array}{cc}
g_{\mu \nu}+A_{\mu} A_{\nu} & A_{\mu} \\
A_{\nu} & 1
\end{array}\right)
$$

where $g_{\mu \nu}=e_{\mu}^{a} e_{\nu}^{b} \eta_{a b}$. It should be remarked that in the present case $e_{(5)}$ is fixed and the $e_{a}$ are only determined up to local 4-d Lorentz transformations that are independent of $\theta$. Inserting the cobasis (4.1) into the action (3.4) we obtain

$$
S=\int_{\mathcal{M} \times S^{1}} \mathrm{~d}^{5} x \sqrt{-\gamma}\left({ }^{(4)} R-\frac{1}{4} F_{\mu \nu} F_{\rho \sigma} g^{\mu \rho} g^{\nu \sigma}\right),
$$

where $F_{\mu \nu} \equiv 2 \partial_{[\mu} A_{\nu]}$. Since $\sqrt{-\gamma}=\sqrt{-g}$ and the integrand does not depend on $\theta$, we can integrate over the internal $S^{1}$ yielding 


$$
S=\int_{\mathcal{M}} \mathrm{d}^{4} x \sqrt{-g}\left({ }^{(4)} R-\frac{1}{4} F_{\mu \nu} F^{\mu \nu}\right)
$$

where $F^{\mu \nu} \equiv F_{\rho \sigma} g^{\mu \rho} g^{\nu \sigma}$. The functional (4.4) is the action of the Einstein-Cartan-Maxwell theory. It should be emphasized that here the field strength is represented by the fifth component of the torsion tensor, $F_{\mu \nu}=T^{(5)}{ }_{\mu \nu}$. Another argument in favor of this interpretation of the field strength was given in Ref. 15] in connection with an analysis of the formulation of the Aharonov-Casher effect.

\section{INCORPORATION OF A SCALAR FIELD}

The cobasis (4.1) is not the most general parameterization of the Kaluza-Klein geometry. We can introduce a scalar field $\phi\left(x^{\mu}\right)$ which leads to a scale factor of the internal $S^{1}$-manifold. Choosing the parameterization $e_{(5)}=e^{-\phi} \partial_{\theta}$, the cobasis is given by

$$
e^{a}=e_{\mu}^{a} \mathrm{~d} x^{\mu}, \quad e^{(5)}=e^{\phi}\left(\mathrm{d} \theta+A_{\mu} \mathrm{d} x^{\mu}\right) .
$$

Inserting (5.1) into the action (3.4), we obtain after dimensional reduction

$$
S=\int_{\mathcal{M}} \mathrm{d}^{4} x \sqrt{-g}\left(e^{\phi(4)} R-\frac{1}{4} e^{3 \phi} F_{\mu \nu} F^{\mu \nu}+2 e^{\phi} \partial_{\mu} \phi T^{\rho}{ }_{\nu \rho} g^{\mu \nu}\right) .
$$

There is an apparent coupling of the scalar field and the torsion tensor described by the last term in the action. However, the scalar curvature ${ }^{(4)} R$ contains a divergence $2 g^{\mu \nu} \stackrel{\circ}{D}_{\mu} T^{\rho}{ }_{\nu \rho}$ which, after a partial integration, cancels the last term exactly. Thus, we obtain

$$
S=\int_{\mathcal{M}} \mathrm{d}^{4} x \sqrt{-g}\left\{e^{\phi}\left[{ }^{(4)} \stackrel{\circ}{R}+g^{\mu \nu}\left(K^{\rho}{ }_{\mu \nu} K^{\sigma}{ }_{\rho \sigma}-K^{\rho}{ }_{\mu \sigma} K^{\sigma}{ }_{\rho \nu}\right)\right]-\frac{e^{3 \phi}}{4} F_{\mu \nu} F^{\mu \nu}\right\},
$$

where

$$
K_{\nu \rho}^{\mu} \equiv \frac{1}{2}\left[T_{\nu \rho}^{\mu}-g^{\mu \sigma}\left(g_{\rho \lambda} T_{\sigma \nu}^{\lambda}+g_{\nu \lambda} T_{\sigma \rho}^{\lambda}\right)\right]
$$

is the 4 -d contortion tensor and ${ }^{(4)} \stackrel{\circ}{R}$ is the scalar curvature of the 4 -d metric $g_{\mu \nu}$. The action (5.3) shows that torsion is not induced by the scalar field; variation with respect to the torsion tensor leads to field equations which imply a vanishing torsion in the absence of spinning matter. This stands in contrast to some other approaches to gravitation with torsion and Brans-Dicke field 16 18] where the torsion acquires a contribution from the Brans-Dicke scalar even in the absence of matter.

The factor $e^{\phi}$ in the gravitational part of the action (5.3) can be removed by introducing a different parameterization of the cobasis. An appropriate form is

$$
\hat{e}^{a}=e^{-\frac{1}{3} \phi} e_{\mu}^{a} \mathrm{~d} x^{\mu}, \quad \hat{e}^{(5)}=e^{\frac{2}{3} \phi}\left(\mathrm{d} \theta+A_{\mu} \mathrm{d} x^{\mu}\right)
$$

corresponding to a Weyl-factor $e^{-\frac{2}{3} \phi}$ in the metric. In this case, the action (3.4) yields

$$
S=\int_{\mathcal{M}} \mathrm{d}^{4} x \sqrt{-g}\left({ }^{(4)} R-\frac{1}{4} e^{2 \phi} F_{\mu \nu} F^{\mu \nu}-\frac{2}{3} \partial_{\mu} \phi \partial_{\nu} \phi g^{\mu \nu}\right) .
$$

\section{DISCUSSION}

In this article, we have put forward a modification of the Kaluza-Klein model which unifies 4-d Einstein-Cartan gravitation with electromagnetism and a scalar field. The main ingredient was the introduction of a geometry, which was called semi-teleparallel geometry, relying on a connection 
that parallelizes the direction of the space-time manifold singled out by a vector field. In a certain sense, we did not introduce new structures since the constraints on the space-time geometry were traced back to the dimensional reduction procedure and to the Killing vector field which already exist in the original Kaluza-Klein unification.

Besides this unification scheme with torsion, there are at least two alternative approaches.

The first approach, which is treated in the literature [四, ], consists in the use of 5-d EinsteinCartan gravitation as starting point. This procedure also leads to the Einstein-Cartan-Maxwell theory. However, there emerge extra fields - the components of the torsion tensor involving the fifth dimension - which have to be interpreted physically. These extra fields vanish in the vacuum by their field equations, but they will play a role when spinning matter is present.

A possible second approach starts from 5-d Einstein-Cartan gravitation where the components of the torsion tensor involving the fifth dimension are set to zero a priori. This method leads to the same results as obtained in this article. However, the geometry of space-time is completely different. Moreover, there is no obvious motivation to constrain a part of the torsion tensor. Generally, the imposition of the constraint of a vanishing torsion tensor is unnatural since it is not connected with symmetry properties - as it is, e.g., in the case of vanishing nonmetricity. In the approach proposed in this article, the torsion is not restricted kinematically; it is the curvature that is constrained, and this follows from a symmetry breaking naturally implied by the dimensional reduction.

The concept of semi-teleparallel geometry may also be applied to the higher dimensional unification of gravitation with Yang-Mills theory. Furthermore, the concept could even be used in 4-d space-time. These generalizations are presently under study.

[1] Th. Kaluza, Sitzungsber. Preuss. Akad. Wiss. Berlin, 966 (1921)

[2] O. Klein, Z. Phys. 37, 895 (1926)

[3] F. W. Hehl, J. D. McCrea, E. W. Mielke and Y. Ne'eman, Phys. Rep. 258, 1 (1995)

[4] M. W. Kalinowski, Int. J. Theoret. Phys. 20, 563 (1981)

[5] G. Germán, Class. Quantum Grav. 2, 455 (1985)

[6] G. Germán, A. Macías and O. Obregón, Class. Quantum Grav. 10, 1045 (1993)

[7] G. M. Zhang and Y. L. Wu, Int. J. Theoret. Phys. 35, 1211 (1996)

[8] D. Schütze, J. Math. Phys. 26, 2596 (1985)

[9] X. J. Lee and Y. L. Wu, Phys. Lett. 165A, 303 (1992)

[10] W. Kopczyński, Acta Phys. Polon. B 10, 365 (1979)

[11] M. O. Katanaev, Theor. Math. Phys. 56, 795 (1984)

[12] M. O. Katanaev and I. V. Volovich, Phys. Lett. 156B, 327 (1985)

[13] N. A. Batakis, Class. Quantum Grav. 3, L99 (1986)

[14] S. Kobayashi and K. Nomizu, Foundations of Differential Geometry (Wiley, New York, 1963) Vol.1

[15] C. Kohler, Phys. Lett. 237A, 195 (1998)

[16] G. Germán, Phys. Rev. D 32, 3307 (1985)

[17] S. -W. Kim, Phys. Rev. D 34, 1011 (1986)

[18] S. -W. Kim and B. H. Cho, Phys. Rev. D 36, 2314 (1987) 